\title{
Microfabricated intracortical extracellular matrix-microelectrodes for improving neural interfaces
}

\author{
Wen Shen ${ }^{1,8}$, Suradip Das², Flavia Vitale ${ }^{3}$, Andrew Richardson², Akshay Ananthakrishnan (1) ${ }^{4}$ Laura A. Struzyna ${ }^{5}$, \\ Daniel P. Brown², Naixin Song ${ }^{6}$, Murari Ramkumar ${ }^{7}$, Timothy Lucas², D. Kacy Cullen², Brian Litt ${ }^{3}$ and Mark G. Allen
}

\begin{abstract}
Intracortical neural microelectrodes, which can directly interface with local neural microcircuits with high spatial and temporal resolution, are critical for neuroscience research, emerging clinical applications, and brain computer interfaces (BCl). However, clinical applications of these devices remain limited mostly by their inability to mitigate inflammatory reactions and support dense neuronal survival at their interfaces. Herein we report the development of microelectrodes primarily composed of extracellular matrix (ECM) proteins, which act as a bio-compatible and an electrochemical interface between the microelectrodes and physiological solution. These ECM-microelectrodes are batch fabricated using a novel combination of micro-transfer-molding and excimer laser micromachining to exhibit final dimensions comparable to those of commercial silicon-based microelectrodes. These are further integrated with a removable insertion stent which aids in intracortical implantation. Results from electrochemical models and in vivo recordings from the rat's cortex indicate that ECM encapsulations have no significant effect on the electrochemical impedance characteristics of ECM-microelectrodes at neurologically relevant frequencies. ECM-microelectrodes are found to support a dense layer of neuronal somata and neurites on the electrode surface with high neuronal viability and exhibited markedly diminished neuroinflammation and glial scarring in early chronic experiments in rats.
\end{abstract}

\section{Introduction}

The capability of intracortical neural microelectrodes to obtain action potentials from local neural microcircuits makes them an indispensable tool for accurate clinical diagnoses and treatment of neurological disorders, neuroscientific electrophysiological research, and applications such as brain computer interfaces $(\mathrm{BCI})^{1,2}$. Silicon or metal-based intracortical neural microelectrodes have demonstrated significant successes in neuronal recording $^{3}$, deep brain stimulation ${ }^{4}$, and neuronally controlled prosthetics to restore lost sensory and motor abilities such

Correspondence: Mark G. Allen (mallen@seas.upenn.edu)

${ }^{1}$ Krishna P. Singh Center for Nanotechnology, University of Pennsylvania, Philadelphia, PA 19104, USA

${ }^{2}$ Department of Neurosurgery, Perelman School of Medicine, University of Pennsylvania, Philadelphia, PA 19104, USA

Full list of author information is available at the end of the article. as robotic arm movement ${ }^{5-7}$, visual function ${ }^{8}$, speech function ${ }^{9}$, etc. However, these inorganic devices also have several shortcomings, including poor biocompatibility and large mechanical mismatch with host neural tissue, which can lead to damage of local neuronal environments, inflammation, and scar formation ${ }^{10-13}$. These issues have driven the investigation of new materials for microelectrodes with emphasis on greater neural compatibility and mechanical flexibility.

It is possible to achieve these complementary requirements by building interfaces out of protein-based composite materials ${ }^{14}$. Type I collagen (collagen I, for short), due to fibrillary structures, provides mechanical support to resident cells in the extracellular matrix $(E C M)^{15,16}$, It has already been used in many applications such as wound healing ${ }^{17}$ and soft tissue repair ${ }^{18}$; however, its application in the brain alone as neural interfaces has not

\section{(c) The Author(s) 2018}

(c) (i) Open Access This article is licensed under a Creative Commons Attribution 4.0 International License, which permits use, sharing, adaptation, distribution and reproduction cc) in any medium or format, as long as you give appropriate credit to the original author(s) and the source, provide a link to the Creative Commons license, and indicate if changes were made. The images or other third party material in this article are included in the article's Creative Commons license, unless indicated otherwise in a credit line to the material. If material is not included in the article's Creative Commons license and your intended use is not permitted by statutory regulation or exceeds the permitted use, you will need to obtain permission directly from the copyright holder. To view a copy of this license, visit http://creativecommons.org/licenses/by/4.0/. 
been demonstrated. Among the major ECM proteins in the brain tissue, fibronectin assists the cell-matrix adhesions through specific peptide sequences ${ }^{19,20}$, laminin and Type IV collagen (collagen IV, for short) not only form two-dimensional networks in basal laminae, but also interact with many cell surface proteins and regulate development, differentiation, and cell migration ${ }^{20,21 .}$

Previously, nanoscale coatings using ECM components such as laminin and fibrin on the surface of silicon- or metal-based neural electrodes have been realized through passive adsorption $^{22}$ and covalent immobilization ${ }^{23}$. These ECM-coated electrodes were reported to have reduced inflammation and supported survival of viable neurons ${ }^{22,23}$, However, these effects only persisted for approximately 1 week, after which the ECM coatings were observed to be dissolved ${ }^{22,23}$, This indicates that merely coating ECM materials onto microelectrodes may be insufficient in mitigating the evolution of the inflammation processes at neural interfaces ${ }^{24}$. Instead, developing microelectrodes which are primarily composed of ECM materials may facilitate sustained stability and biocompatibility of microelectrode interfaces.

Although ECM-mimics have been fabricated using micro-patterning approaches including coating, molding, electrospinning, and wet-spinning ${ }^{25}$, the production of stand-alone, natural materials-based microelectronic devices with sizes in the range of tens to hundreds of micrometers (in the size range of neurons) remains to be achieved $^{25-27}$. To address this apparent gap, we have developed ECM composites that are predominantly comprised of collagen I and functional components of the brain tissue, such as, laminin, fibronectin, and collagen IV. As is the case with most natural tissues, the fibrillar structure of collagen I plays a key role in providing structural support to the ECM-encapsulated microelectrodes developed in this study, while the functional components provide biological cues to regulate cellular adhesion and potentially further depress inflammatory responses at the neural interfaces. We have developed a batch microfabrication technology, which can enable these thick ECM composites to effective encapsulate the recording components of ECMmicroelectrodes and facilitate their integration with removable insertion stents. Using this rapid fabrication approach, ECM-microelectrodes with sizes comparable to commercially available Si-microelectrodes (Michigan M15, NeuroNexus Technologies, Inc., Ann Arbor, MI, USA) have been produced. Their biocompatibility and interface stability have been comprehensively evaluated in vitro for neural culture viability, neuronal adhesion, growth, and network formation. Unlike laminin, fibronectin, and collagen IV, collagen I is not a natural constituent of the ECM found in brain tissues. Therefore, the local host cell/tissue responses to ECM-microelectrodes composed of collagen I (collagen I microelectrodes) post implantation have been evaluated.
In addition, the influences of ECM materials on the electrochemical performance of the ECM-microelectrodes are studied using electrochemical impedance spectroscopy (EIS) and described using new circuit models. Their neural recording capability has been demonstrated in vivo in the rat cortex region.

\section{Results \\ Microfabricated ECM-microelectrodes}

Micro-transfer-molding has been demonstrated as an appropriate bottom-up approach to fabricate various microstructures from natural materials ${ }^{28,29}$, and to integrate these materials with microelectronic devices ${ }^{30}$. In addition, excimer laser micromachining has been used as an effective top-down rapid production technique to produce microstructures in natural materials ${ }^{31}$. Bringing together these technologies to bear on natural products such as ECM materials, we created implantable ECMmicroelectrodes integrated with insertion stents at high throughput, while preserving the biological nature of these materials. The insertion stents can help minimize damages due to implantation by (a) minimizing the required insertion force and (b) allowing for smaller device footprint ${ }^{32}$.

Figure 1a, b schematically illustrates the key processes involved in the fabrication of ECM-microelectrodes integrated with removable insertion stents. The ECMmicroelectrodes comprise the functional $\mathrm{Au}$-Parylene $\mathrm{C}$ (Px) (pre-ECM) microelectrode with the ECM encapsulation layers, serving as bio-compatible neural interfaces. Four types of ECM-microelectrodes: collagen I microelectrodes, collagen I/collagen IV microelectrodes, collagen I/fibronectin microelectrodes, and collagen I/laminin microelectrodes, each with dimensions $(100 \mu \mathrm{m}$ in width, $30 \mu \mathrm{m}$ in thickness, and $5 \mathrm{~mm}$ in length) comparable to those of commercial silicon M15-microelectrodes (5 mm in length, $80 \mu \mathrm{m}$ in width at the tip and $300 \mu \mathrm{m}$ in width at the base, and thickness of $15 \mu \mathrm{m}$ ), were realized using these microfabrication techniques. The collagen I microelectrode is composed of collagen I, while the latter three are composed of ECM composites of collagen I (as structural support) and brain ECM proteins including collagen IV, fibronectin, and laminin. These ECM encapsulations provided adequate mechanical strength required during the fabrication processes. In addition, the long-fibrous morphology of collagen I prepared in this study (Fig. 1f, i) exhibited dimensions (sub-micrometer in diameter and tens of micrometers in length) similar to those of native collagen fibers ${ }^{33}$, thereby simulating a more in vivo-like microenvironment ${ }^{34}$. To aid in insertion, ECM-microelectrodes were physically adhered to insertion stents fabricated from $25-\mu$ m-thick stainless steel (SS); as will be described below, this physical adhesion degraded upon post-insertion exposure to wet environments allowing selective withdrawal of the insertion stent. 


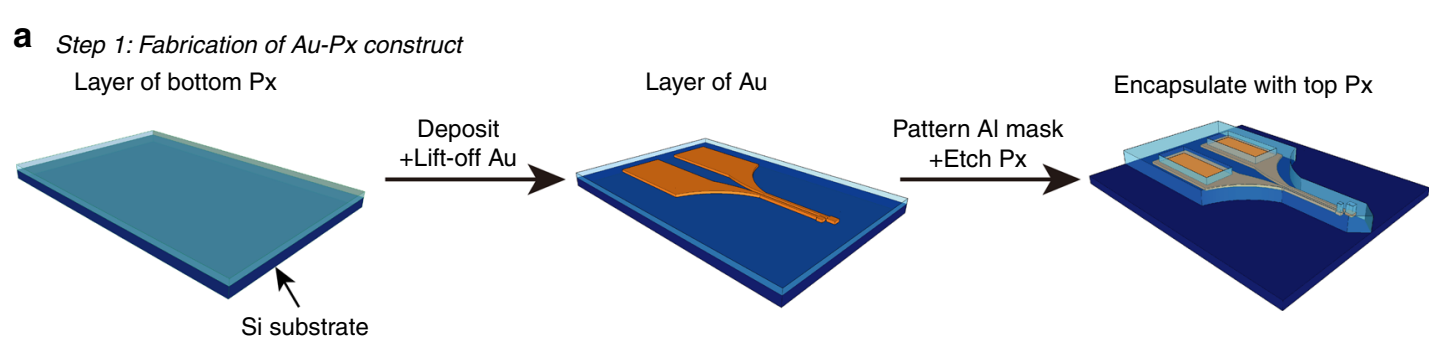

b Step 2: Fabrication of integrated ECM-neural microelectrodes Layer of Top ECM

Encapsulate with bottom ECM

Transfer onto SS stent

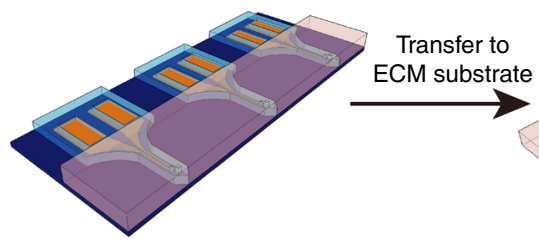

d

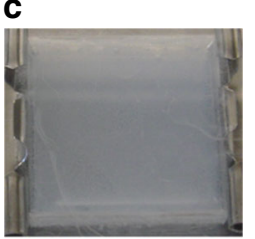

$\bar{f}$

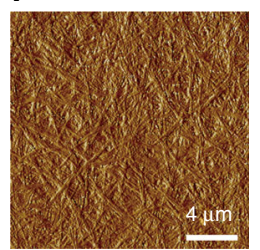

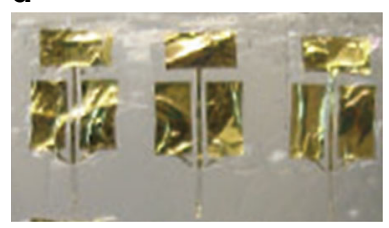

g

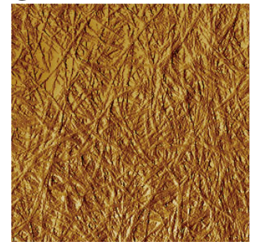

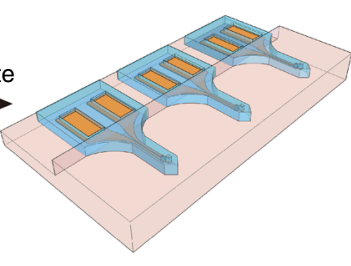

e

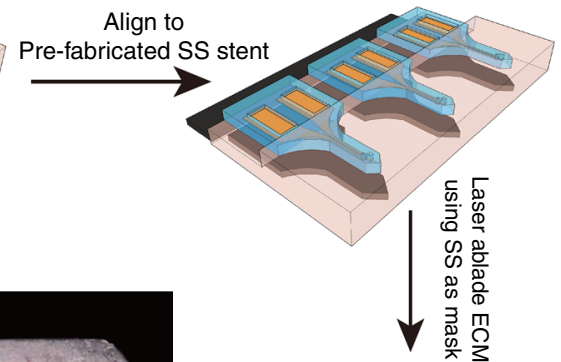

Integrated ECM-microelectrodes

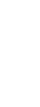
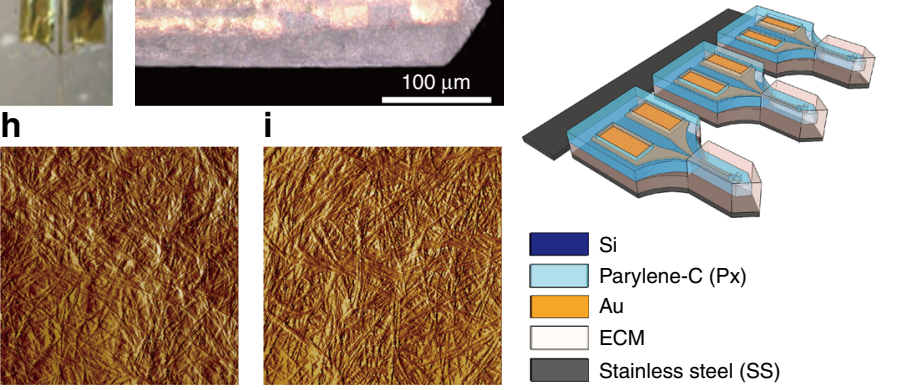

Fig. 1 Schematic illustration and images corresponding to steps for fabricating ECM- microelectrodes integrated with insertion stents. a Steps for fabricating Au-Px construct, and $\mathbf{b}$ transfer them onto ECM substrate, integrate with SS stent, and ablate the using excimer laser. Images showing c ECM hydrogel (bulk), d ECM-microelectrodes, e tip and recording sites of an ECM-microelectrode (scale bar: $100 \mu$ m); AFM images of ECM substrates: $\mathbf{f}$ collagen I, $\mathbf{g}$ collagen I/collagen IV, h collagen I/fibronectin, $\mathbf{i}$ collagen I/laminin

The tensile and bending stiffnesses of these SS-integrated ECM-microelectrodes were calculated to be $\sim 56.3 \mathrm{kN} \mathrm{m}^{-1}$ and $\sim 2.20 \mathrm{kN} \mathrm{m}^{-1}$, respectively. These are comparable to those of conventional silicon-based microelectrodes, $\sim 151 \mathrm{kN} \mathrm{m}^{-1}$ and $\sim 2.13 \mathrm{kN} \mathrm{m}^{-1}$ (ref. ${ }^{35}$ ), respectively. This enabled insertion of the otherwise flexible ECMmicroelectrodes into the desired depth. Testing of the insertion mechanism in vitro using a brain phantom (1\% agarose gel) was performed to ensure (i) no premature detachment of the ECM electrode from the insertion stent during the insertion process; (ii) successful detachment and withdrawal of the insertion stent upon completion of insertion; and (iii) preserved functionality of the ECM electrode as assessed by electrical impedance spectroscopy (EIS). For both these in vitro and subsequent in vivo implantations, an insertion speed of approximately $2 \mathrm{~mm} \mathrm{~s}^{-1}$ was used, which resulted in a total insertion time of approximately $1 \mathrm{~s}$. No premature detachment was observed, indicating that the adhesion between the surfaces of the SS stent and dry ECM-microelectrodes was sufficient to withstand the effect of shear forces that arose during implantation. It is likely that this insertion time is much less than the time required for fluid to diffuse from the host tissue to the SS stent-ECM microelectrode interface, reducing the risk of premature separation during insertion. Within $5 \mathrm{~min}$ post-insertion, sufficient hydration of the ECM materials was observed, separating the ECM-microelectrodes from the insertion stent. The insertion stent could then be removed, thereby leaving the ECM-microelectrodes implanted. Functionality of the ECM-electrodes implanted using this technique is discussed below.

\section{Impedance characterization of ECM-microelectrodes}

As the neural recording functionality of the microelectrodes typically relies on their electrochemical impedance 

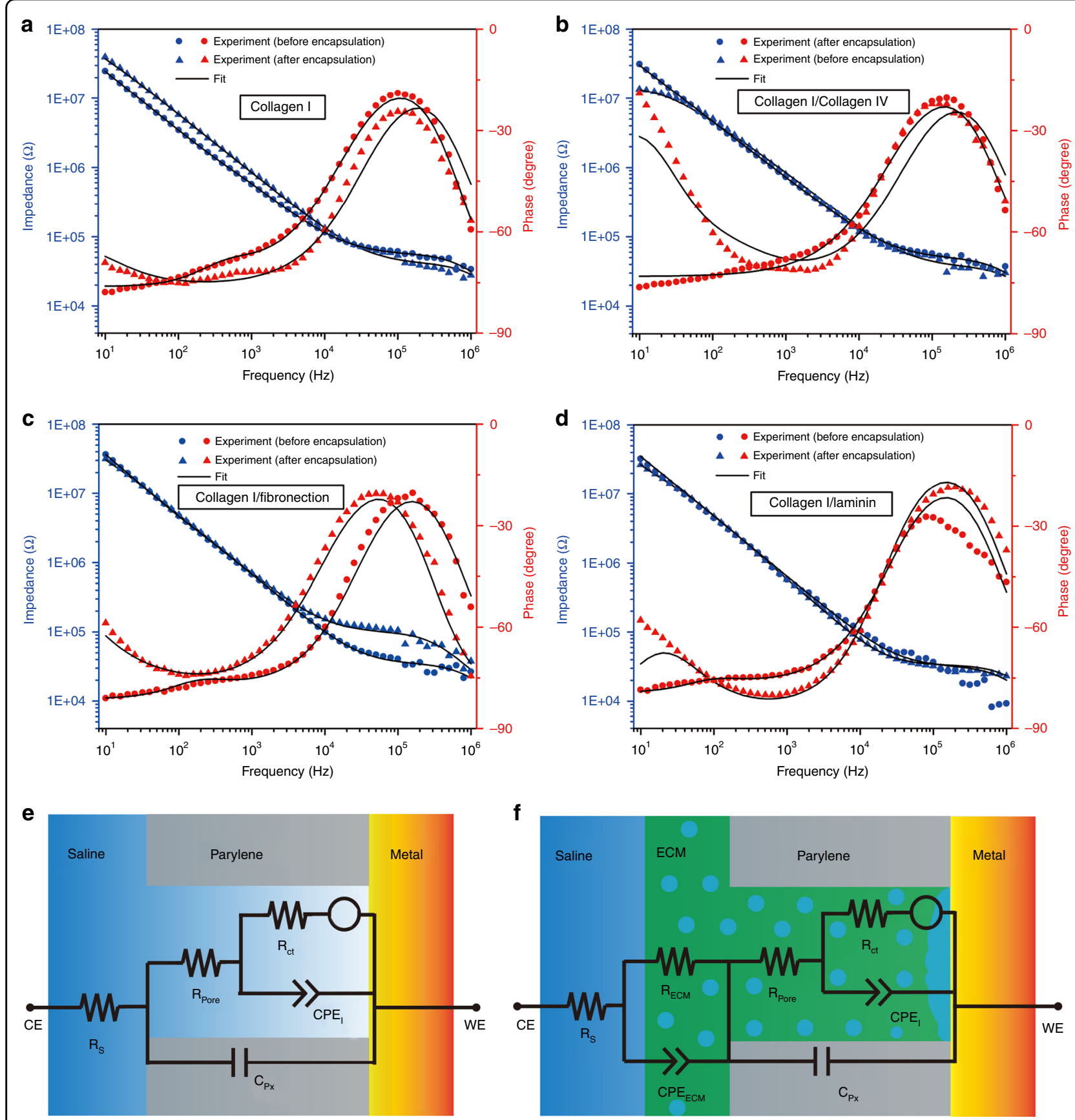

Fig. 2 Electrochemical impedance of ECM-microelectrodes. a-d Bode plot for a collagen I microelectrode, $\mathbf{b}$ collagen I/collagen IV microelectrode, c collagen I/fibrinectin microelectrode, and $\mathbf{d}$ collagen I/laminin microelectrode. Measurement results are shown with symbols and fitting results are shown with solid lines. e-f Physical representation of the equivalent circuit models for e pre-ECM-microelectrodes and f ECM-microelectrodes

characteristics, EIS measurements were performed on ECM-microelectrodes as well as corresponding pre-ECMmicroelectrodes in order to understand the influences of ECM encapsulations on the electrochemical impedance characteristics of the neural microelectrodes. For each electrode tested, after ten sequential runs, no degradation in
EIS performance was observed. Fig. 2a-d shows magnitude and phase behavior of electrochemical impedances as a function of frequency for the various ECM encapsulations considered in this study. In all cases, the magnitude of impedance was found to decrease monotonically with frequency and its value at the recording frequency of $1 \mathrm{kHz}$ 
was found to vary between $550 \mathrm{k} \Omega$ and $900 \mathrm{k} \Omega$, which are suitable for extracellular recording ${ }^{36-38}$. In comparison to the impedance magnitude, the observed trend of phase angle vs. frequency was more complex. Also, from Fig. 2a-d, it can be seen that the phase behavior of ECMmicroelectrodes is noticeably different from its corresponding pre-ECM counterpart.

\section{Model description}

These trends in electrochemical responses were related to the electrical properties of constituent electrode materials by fitting the impedance data to equivalent circuit models shown in Fig. 2e, f. While the overall circuit architecture of these models bears some similarity to that proposed recently by Shi et al. ${ }^{39}$, they differ in their application, both in terms of the phenomena being modeled as well as the design of electrodes and materials employed. The circuit elements in Fig. 2e, f are hereby described in reference to the physical phenomena that they model. The capacitor, $C_{\mathrm{Px}}$, reflects the dielectric properties of the Px layers and the resistor, $R_{\text {pore }}$, quantifies the resistance to the motion of ions within the opening in the uppermost Px layer that leads up to the $\mathrm{Au}$-electrolyte interface. A Kovacs circuit was adopted to model the Au-electrolyte interface as a constant phase element, $C P E_{\mathrm{I}}$, representing the interfacial capacitance, in parallel with a series combination of charge transfer resistance, $R_{\mathrm{ct}}$, and diffusion-based semi-infinite Warburg element, $W$. The Warburg element, $W$, accounts for impedance arising from ion diffusion, while $R_{\mathrm{ct}}$ describes the impedance to Faradaic reactions. We model the effects of porous ECM encapsulation through an ad-hoc circuit, comprising a parallel arrangement of constant phase element, $C P E_{\mathrm{ECM}}$, and a resistor, $R_{\mathrm{ECM}}$. Here, $C P E_{\mathrm{ECM}}$ describes the dielectric properties of the ECM encapsulation while $R_{\mathrm{ECM}}$ represents the resistance to movement of ions through its pores.

\section{Results from model fit}

To obtain the parametric values of these circuit elements (except $R_{\mathrm{ct}}$ ), we fit the impedance characteristics of preECM and ECM-microelectrodes, which are presented in Fig. 2a and d, to the circuit models shown in Fig. 2e, f, respectively. This was performed using Echem Analyst, a proprietary software developed by Gamry. We estimated $R_{\mathrm{ct}}$ via cyclic voltammetry, from the slope of the current vs. overpotential plot (Supplementary Figure S1) using the low field approximation to the Butler-Volmer equation. Thereby $R_{\mathrm{ct}}$ was determined to be $3.6 \mathrm{M} \Omega$, and was fixed at this value while fitting models. The fitting routine used in this study followed a two-step procedure. First, the measured impedance data of pre-ECM electrodes were fit to the circuit shown in Fig. 2e and the values of corresponding circuit elements were determined. Of these, $C_{\mathrm{Px}}, C P E_{\mathrm{I}}$, and $R_{\mathrm{s}}$ were assumed to be largely unaffected by subsequent ECM encapsulation. Next, the impedance data of ECMmicroelectrodes were fit to the circuit model shown in Fig. $2 \mathrm{f}$ using exactly these values of $C_{\mathrm{Px}}, C P E_{\mathrm{I}}$, and $R_{\mathrm{s}}$ to determine the values of remaining circuit elements.

Results obtained from this fitting routine for both preECM and the corresponding ECM-microelectrode samples are illustrated by solid lines in Fig. 2a-d. Also, parametric values corresponding to circuit elements comprising Fig. 2e, f are summarized in Supplementary Table S1 and Table 1. $C P E_{\mathrm{ECM}}$ and $R_{\mathrm{ECM}}$ for various ECM encapsulations were found to typically lie in ranges of $(1.82-28.7) \times 10^{-10} \mathrm{~S} \times s^{n}\left(n_{\mathrm{ECM}}\right.$ varied in a range of $0.88-1)$ and $5-21 \mathrm{M} \Omega$, respectively. Overall, the impedance values of ECM-microelectrodes at $1 \mathrm{kHz}$ showed a moderate increase from their corresponding pre-ECM electrodes, as summarized in Table 1.

\section{ECM-microelectrodes enhanced neuronal viability and neurite outgrowth}

The cytocompatibility of ECM-microelectrodes was assessed based on their ability to support the growth and viability of primary cerebral cortical neurons in vitro. Here, neurons were cultured directly on pre-ECM-microelectrodes (negative control) and ECM-microelectrodes comprising collagen I, collagen I/fibronectin, collagen I/laminin, or collagen I/collagen IV, with comparison to neurons grown in planar culture on polystyrene coated with poly-D-lysine + laminin (positive control). Neuronal viability was poor on pre-ECM-microelectrodes or collagen I microelectrode, but was improved with the addition of fibronectin, laminin, or collagen IV (Fig. 3). In particular, neurons grown on ECM-microelectrodes with additional laminin or collagen IV revealed a markedly increased area of viable cells as compared to pre-ECM-microelectrodes or collagen I microelectrodes. Subsequent calculation of the culture viability ratio revealed that all of the ECM-microelectrodes had significantly higher cell viability as compared to the preECM-microelectrodes group $(p<0.05)$ (Fig. 3f). Out of the experimental ECM-microelectrode groups, a maximum mean culture viability of $70.6 \%$ was found for the collagen I/collagen IV microelectrode group, which was comparable to the viability observed for the planar control cultures (74.7\%). Further, the skeletal framework of all the electrodes exhibited blue autofluorescence which can be attributed to the presence of parylene ${ }^{40}$.

In addition, immunocytochemistry was performed using a neuronal cytoskeletal marker ( $\beta$-tubulin III) to assess neurite outgrowth and network formation on the ECMmicroelectrodes. Consistent with the viability assay, we found that the pre-ECM-microelectrode and the collagen I microelectrode groups exhibited minimal cell adherence on the microelectrode surface, as the $\mathrm{Px}$ and $\mathrm{Au}$ structures were completely visible (Fig. 4a, b). However, 
Table 1 Summary of the EIS measurement and fitted parameters of the ECM-microelectrodes

\begin{tabular}{|c|c|c|c|c|c|}
\hline & & \multicolumn{4}{|c|}{ ECM-microelectrodes } \\
\hline & & Collagen I & Collagen I/Collagen IV & Collagen I/Fibronectin & Collagen I/Laminin \\
\hline \multirow[t]{2}{*}{$C P E_{1}\left(10^{-10} S \times s^{n}\right)$} & $Q_{1}$ & 11.8 & 11.5 & 7.78 & 9.07 \\
\hline & $n_{1}$ & 0.83 & 0.81 & 0.86 & 0.85 \\
\hline$R_{\mathrm{ct}}(\mathrm{M} \Omega)$ & & 3.60 & 3.60 & 3.60 & 3.60 \\
\hline$W\left(10^{-9} S \times s^{1 / 2}\right)$ & & 1.92 & 15.3 & 3.41 & 1.56 \\
\hline$C_{P x}(p F)$ & & 4.12 & 4.14 & 5.84 & 5.44 \\
\hline$R_{\mathrm{s}}(\mathrm{k} \Omega)$ & & 1.39 & 1.90 & 1.65 & 1.42 \\
\hline$R_{\text {pore }}(\mathrm{k} \Omega)$ & & 39.5 & 47.5 & 91.1 & 99.6 \\
\hline \multirow[t]{2}{*}{$C P E_{\mathrm{ECM}}\left(10^{-10} S \times s^{n}\right)$} & $Q_{E C M}$ & 1.82 & 8.55 & 2.39 & 28.7 \\
\hline & $n_{\mathrm{ECM}}$ & 0.88 & 1.00 & 1.00 & 0.96 \\
\hline$R_{E C M}(\mathrm{M} \Omega)$ & & 20.5 & 5.81 & 6.34 & 6.38 \\
\hline$|Z|_{\text {pre-ECM }}(\mathrm{k} \Omega)$ & & 575 & 732 & 674 & 581 \\
\hline$|Z|_{\text {post-ECM }}(\mathrm{k} \Omega)$ & & 852 & 791 & 707 & 583 \\
\hline
\end{tabular}

ECM-microelectrodes with fibronectin, laminin, or collagen IV were found to have a dense layer of neuronal somata and neurites on the microelectrode surface, as shown by significant expression of $\beta$-tubulin III (Fig. 4c, e). Similar neural network growth was observed in twodimensional (2D) planar cultures, although the network topography was more homogeneous likely due to the uniform geometry in planar culture (Fig. 4f). These findings demonstrate the cytocompatibility of the ECMmicroelectrodes and their ability to support the survival and growth of primary cortical neurons with the additional application of fibronectin, laminin, or collagen IV.

\section{ECM-microelectrodes reduce the foreign body response}

The neuropathological assessment of the local host cell/ tissue responses to implanted collagen I microelectrodes was performed, using a commercially available siliconbased microelectrode as a control. The presence of the stiff insertion stent facilitated precise intracortical implantation of the collagen I microelectrodes. The subhundred micrometer size augmented intracortical assimilation of the electrode in rodents. At 1-month post implant into the rat brain, tissue sections orthogonal to the trajectory of the microelectrodes were examined using a panel of immunohistochemical markers and highresolution confocal microscopy. In particular, we assessed localized reactive astrocytosis, microglial/macrophage recruitment and activation, general cell recruitment and density, and neuronal/axonal loss in the vicinity of the microelectrodes (Fig. 5). An exacerbated neuroinflammatory response to silicon-based control microelectrodes in comparison to collagen I microelectrodes was observed. For example, at more superficial depths $(2 \mathrm{~mm}$ below the cerebral cortical surface), silicon-based microelectrodes induced a greater astrogliotic response (based on GFAP immunoreactivity and hypertrophy) both at the tissue-microelectrode interface as well as in the general vicinity of the microelectrodes. Here, there was an apparent loss of astrocyte domain organization as there was an increase in (a) astrocyte density and (b) thick hypertrophic somata/processes extending toward and surrounding the silicone microelectrodes. Astrocytes near the collagen I microelectrodes generally presented finer, non-reactive processes and appeared to maintain unique domain organization. Also at superficial depths, exacerbated microgliosis (based on intensity and extent of Iba1 immunoreactivity) was observed around the silicon-based microelectrodes compared to the collagen I microelectrodes. Although most microglia did not appear to be phenotypically reactive (i.e., ameboid) in either group, there was a greater density of Iba1+ cells infiltrating toward the silicon-based microelectrodes relative to the collagen I microelectrodes. Interestingly, these differences in astrocytosis and microgliosis mostly subsided at greater depths, as peri-electrode glial density was relatively similar for each microelectrode by approximately $4 \mathrm{~mm}$ below the cortical surface (Fig. 5). In addition to assessing glial changes, we also examined axonal density and integrity, which revealed no differences in neurofilament staining in the vicinity of the microelectrodes for the two groups. However, the most remarkable difference between animals implanted with silicon-based microelectrodes and collagen I microelectrodes was the total cell density near the microelectrodes in general, and directly at the 

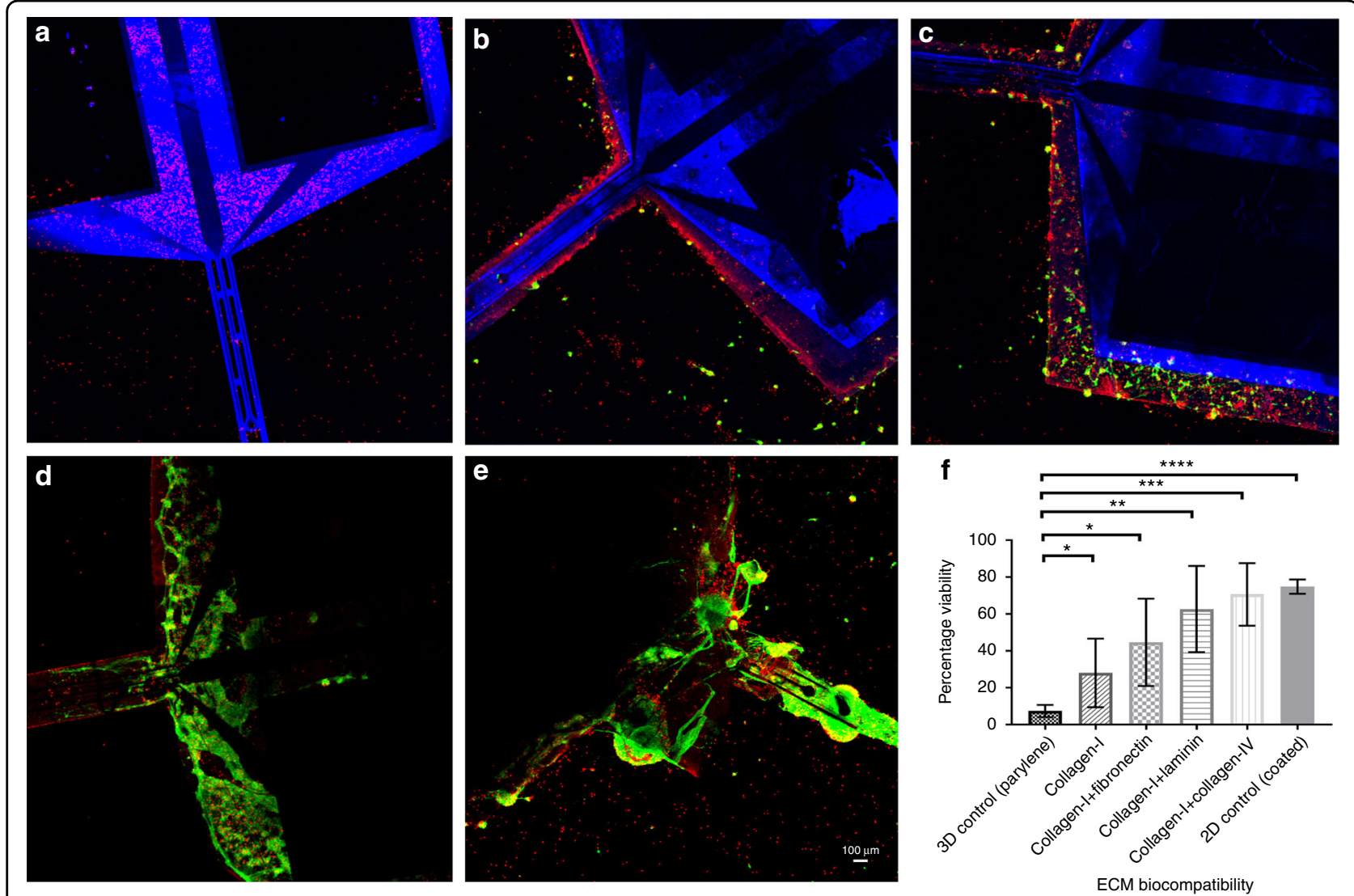

Fig. 3 In vitro biocompatibility of ECM-microelectrodes. Live-dead assay of cortical neurons stained with calcein-AM (green) and ethidium homodimer (red) after 7DIV culture on electrodes encapsulated with a parylene, $\mathbf{b}$ collagen I, c collagen I/fibronectin, $\mathbf{d}$ collagen I/laminin, and e collagen I/collagen IV. Scale bar $=100 \mu \mathrm{m}$. f Percentage viability of cells grown over neural electrodes. Unpaired $t$-test was performed on all the groups in comparison with parylene-coated electrodes; $\left.p<0.05{ }^{*}\right)$

microelectrode-tissue interface in particular. Specifically, we observed a dense layer of host cells lining the siliconbased microelectrodes that appeared consistent with a glial scar. However, the cellular constituents of this dense cell layer only partially labeled for Iba1 and GFAP, suggesting these may predominantly be other infiltrating cell types associated with the glial scar such as endothelial cells and fibroblasts. Of note, this dense cell layer was virtually absent from the interface with the collagen I microelectrodes across all depths examined. These findings demonstrate a differential foreign body response between collagen I microelectrodes and silicon-based microelectrodes at 1-month post-implant, with collagen I microelectrodes eliciting a markedly diminished neuroinflammatory and glial scarring response.

\section{In vivo neural recording}

To verify the recording functionality of the ECM-microelectrodes, measurements were made using collagen I microelectrodes implanted into the cortex of an anesthetized rat. Fig. 6a and c provides examples of local field potentials (LFP, $0.1-300 \mathrm{~Hz}$ ) recorded on a collagen I microelectrode. The recordings show slow oscillations, at approximately $1.5 \mathrm{~Hz}$, typical of the anesthetized state. Consistently, the power spectra show a clear physiological peak at $0.5-3 \mathrm{~Hz}$ (Fig. 6b). Next, we isolated neuronal action potential activity by filtering the data in the spike-band frequency $(\mathrm{SBF}, 300-5000 \mathrm{~Hz})$. The root-mean-square of the SBF data, referred to as the multiunit activity envelope $\left(\mathrm{MUA}_{\mathrm{E}}\right)$, showed substantial modulation. This result demonstrates that the collagen I microelectrodes could record aggregate neuronal activity intracortically (Fig. 6c). Finally, we performed another experiment in a different pair of anesthetized rats to determine whether the collagen I microelectrode conferred any functional benefit over uncoated microelectrodes. A collagen I microelectrode or a commercial silicon microelectrode (NeuroNexus Technologies) was implanted into the whisker sensory cortex of the two rats. A bipolar stimulating electrode was implanted into the homologous area of the opposite brain hemisphere. Biphasic stimulus pulses $\left(100 \mu \mathrm{A}, 0.2 \mathrm{~ms}\right.$ phase $\left.^{-1}\right)$ were delivered and the resulting transcallosal evoked potential 


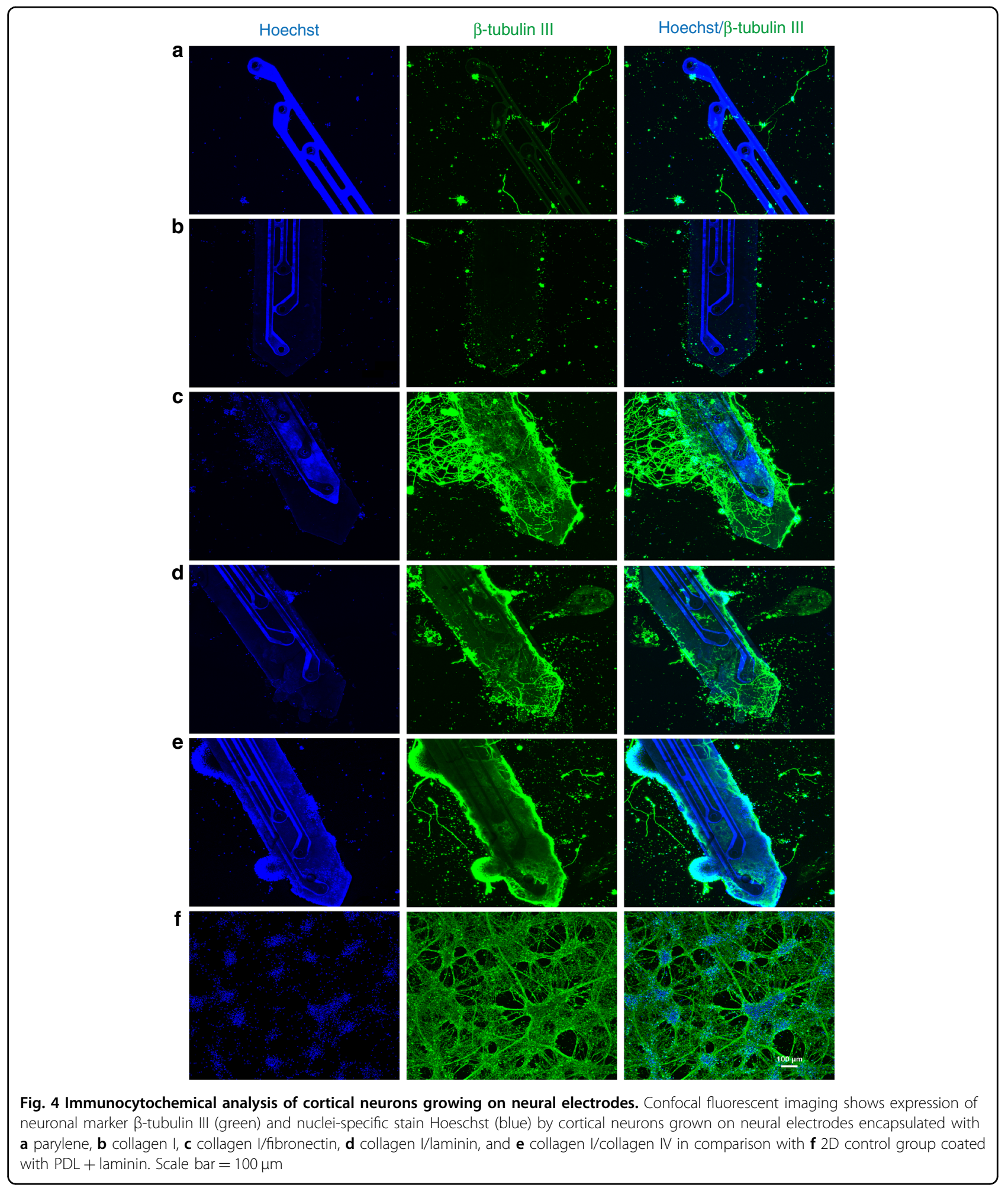

(EP) was recorded (Fig. 6e). The signal-to-noise ratio of EP was in the range of 10-15 for both ECM-electrodes and silicon electrodes and did not significantly differ $(t$-test, $p>0.05$ ) (Fig. 6f). Thus, the ECM encapsulation did not affect the recording performance of the electrodes acutely. We expect the beneficial effects of the ECM encapsulation will manifest over longer time scales. We are currently working to assess these chronic effects. 


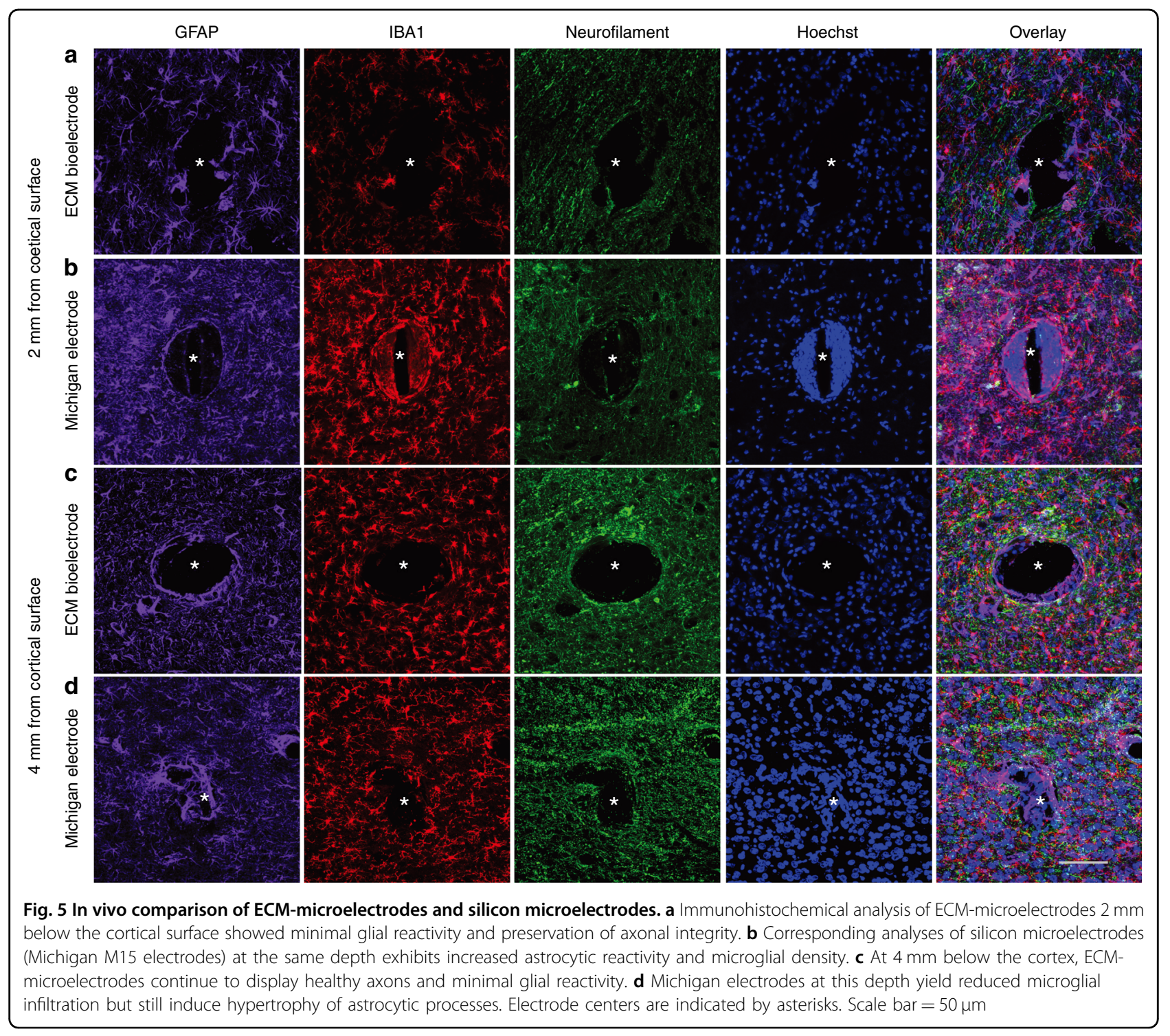

\section{Discussion}

The unique biocompatibility of ECM-microelectrodes provides major advantages for neural interfaces over existing devices based on synthetic materials. However, the production of these devices with footprint in micrometer sizes has been a technical challenge due to the incompatibility of the ECM materials with conventional microfabrication methods. The fabrication techniques presented in this work enabled the realization of implantable ECM-microelectrodes with dimensions that are comparable to commercial Si microelectrodes. These techniques allowed batch fabrication of tens of ECMmicroelectrodes, with potential for high volume scale-up at a lower cost. Moreover, the same fabrication process can be utilized to produce larger arrays of ECMmicroelectrodes with greater number of electrodes. The recording components of these ECM-microelectrodes are effectively encapsulated with thick ECM composites that serve as a biocompatible and electrochemical interface between the microelectrodes and the neural environment.

\section{Validation of equivalent circuit model}

The ECM-microelectrodes possess suitable electrochemical impedance characteristics for neural recordings, which can be analytically described using the equivalent circuit model proposed in this study. From Fig. 2a-d, it can be seen that the proposed circuit models faithfully describe the electrochemical responses of both pre-ECM as well as ECM-microelectrodes over a wide range of frequencies. The fact that this can be achieved by deactivating or activating the ad-hoc circuit shown in Fig. 2f, while retaining identical values 
a

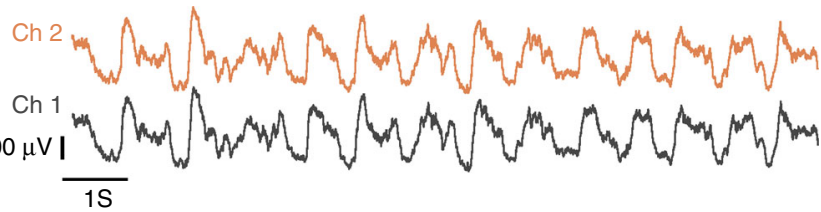

C

C

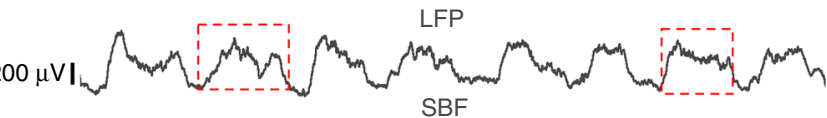

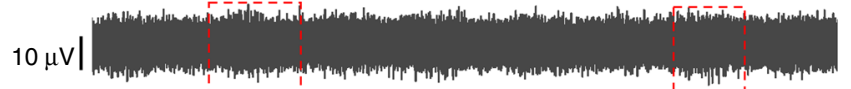

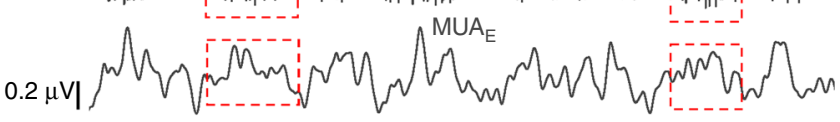
1S

d

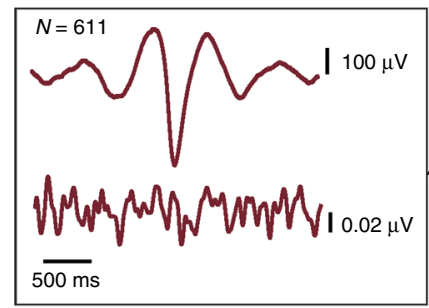

b

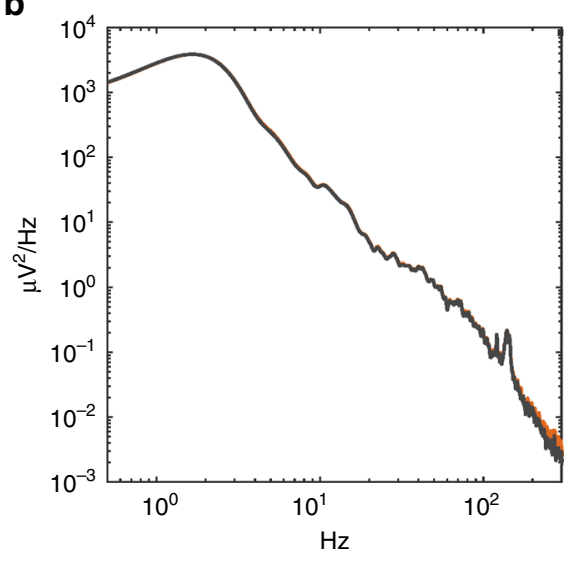

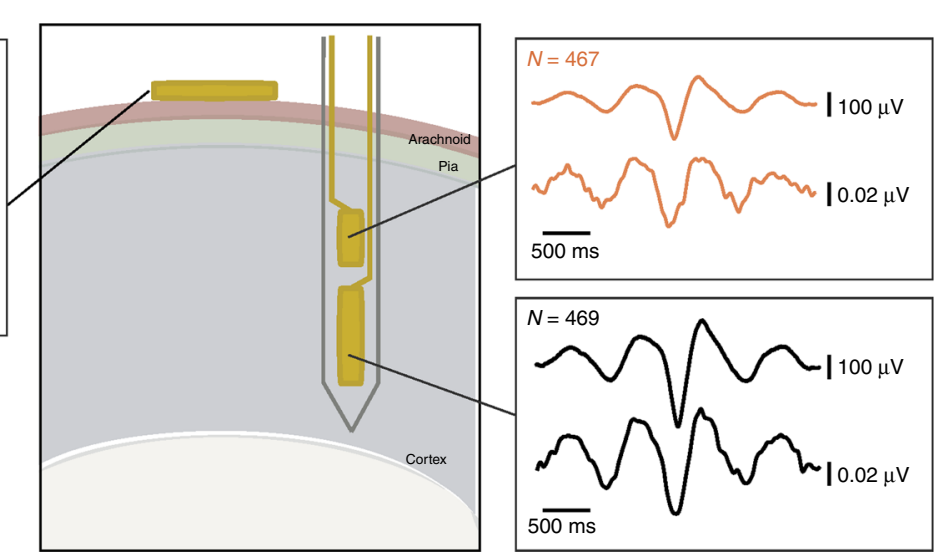

e

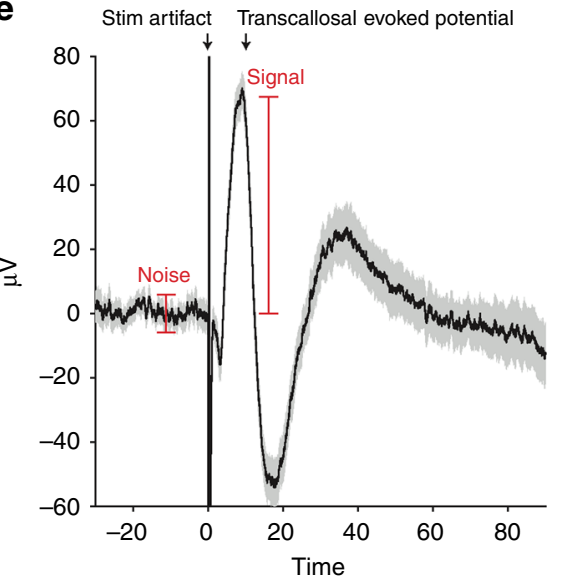

f

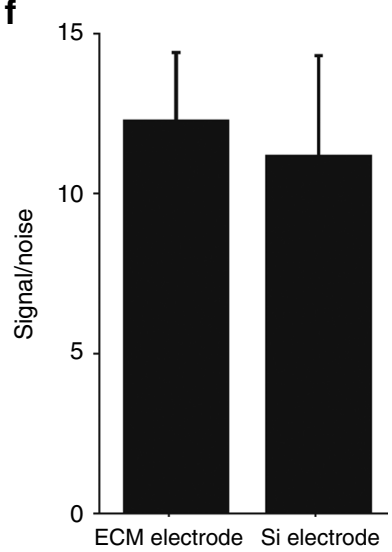

Fig. 6 In vivo recording of spontaneous and evoked neural activity with ECM-microelectrodes in the rat cortex. a Representative 10-s segments of the local field potential (LFP) recordings from the two channels spaced $190 \mu \mathrm{m}$ apart. b Power spectral density of the neural recordings. c Representative 6-s segments of LFP (top), spike-band filtered signal (SBF, middle), and MUA (multiunit activity envelope, bottom) from Channel 2 (electrode close to the tip). The MUAE was obtained from full-wave rectification and $500 \mathrm{~Hz}$ low-pass filtering of the SBF. $\mathbf{d}$ Schematic representation of the surgical placement of the ECM-intracortical microelectrode and micro-electrocorticography (microECoG) surface electrodes. Side panels show cycle-triggered averages (CTAs) of LFP (top trace) and MUA (bottom trace) centered on the trough of the down states of the slow (1-2 Hz) LFP oscillation. e Example transcallosal evoked potential (EP) recorded by the ECM-microelectrode in response to microstimulation of the opposite brain hemisphere. The signal-to-noise ratio (SNR) of the EP was quantified as indicated. $\mathbf{f}$ SNR of the EP recorded by the ECM-microelectrode and by a silicon microelectrode (mean \pm standard deviation). The difference in mean SNR was not significant (unpaired $t$-test, $p>0.05$ ) 
of $C_{\mathrm{Px}}, C P E_{\mathrm{I}}$, and $R_{\mathrm{s}}$ confirms that the model can capture the underlying physics. Further, the value $C_{\mathrm{Px}}$ and the impedances due to $C P E_{\mathrm{I}}$ predicted by the EIS model were also compared to their theoretical estimates. For $C_{\mathrm{Px}}$, a COMSOL simulation of the pre-ECM-microelectrode in phosphate buffered saline (PBS) (conductivity of $1.4 \mathrm{~S} \mathrm{~m}^{-1}$ (ref. $\left.{ }^{41}\right)$ ) was performed and a value of $1 \mathrm{pF}$ was obtained. This corresponds reasonably well with the model prediction of $4.93 \pm 0.95 \mathrm{pF}$. For $C P E_{\mathrm{I}}$, the parametric values, $Q_{\mathrm{I}}$ and $n_{\mathrm{I}}$, corresponding to various pre-ECM electrodes listed in Table 1 were used to calculate the impedance magnitude, $Z_{\mathrm{CPE}-\mathrm{I}, \mathrm{EIS}}$, at $\omega=1 \frac{\mathrm{rad}}{\mathrm{s}}$. This value was found to be $1.03 \pm 0.02 \mathrm{G} \Omega$. To determine its corresponding theoretical value, $Z_{\mathrm{CPE}-\mathrm{I}, \mathrm{th}}$, at $\omega=1 \frac{\mathrm{rad}}{\mathrm{s}}$, Eq. 1 was used to calculate the interfacial capacitance, in accordance with the Gouy-Chapman-Stern model ${ }^{42}$.

$$
\begin{aligned}
& Z_{\mathrm{CPE}-\mathrm{I}, \mathrm{th}}\left(\omega=1 \frac{\mathrm{rad}}{s}\right)=\frac{1}{C_{I}} \\
= & \frac{d_{\mathrm{OHP}}}{A \epsilon_{0} \epsilon_{\mathrm{r}}}+\frac{L_{\mathrm{D}}}{A \epsilon_{0} \epsilon_{\mathrm{r}} \cosh \left(\frac{z V_{\mathrm{OHP}}}{2 V_{\mathrm{t}}}\right)} .
\end{aligned}
$$

Here, $\epsilon_{0}$ is the permittivity of free space, $\epsilon_{\mathrm{r}}$ is the relative permittivity of the double layer, $d_{\mathrm{OHP}}$ quantifies the thickness of the outer Helmholtz plane (OHP), $z$ is the ionic charge in the solution, and $A$ is the exposed area of the recording site. Also, $V_{\mathrm{OHP}}$ is the potential at $\mathrm{OHP}$, while $V_{\mathrm{t}}$ and $L_{\mathrm{d}}$, represent thermal voltage and Debye length, respectively. These were determined through Eqs. $2-4$ (ref. ${ }^{42}$ ):

$$
\begin{aligned}
V_{\mathrm{OHP}} & =V_{0}-\left(\sqrt{\frac{8 q V_{\mathrm{t}} n_{0}}{\epsilon_{0} \epsilon_{\mathrm{r}}}}\right)\left(d_{\mathrm{OHP}} \sinh \left(\frac{z V_{\mathrm{OHP}}}{2 V_{\mathrm{t}}}\right)\right), \\
V_{\mathrm{t}} & =\frac{k T}{q}, \\
L_{\mathrm{D}} & =\sqrt{\frac{\epsilon_{0} \epsilon_{\mathrm{r}} V_{\mathrm{t}}}{2 n_{0} z^{2} q}} .
\end{aligned}
$$

Here, $V_{0}$ is the applied electrode potential, $n_{0}$ is the concentration of ions in the bulk of the solution, $k$ is the Boltzmann constant, and $q$ is the elementary charge. The values corresponding to remaining constants that comprise Eqs. 1-4 are listed in Table S2 in the supplementary information.

In using Eqs. 1-4, PBS was assumed to be a symmetric 1:1 electrolyte comprised of $\mathrm{NaCl}(137 \mathrm{mM})$ and $\mathrm{KCl}$ $(2.7 \mathrm{mM})$. Although this approximation is inexact, due to the presence of phosphate buffer, it provides a resonably accurate estimate of $Z_{\mathrm{CPE}-\mathrm{I}, \mathrm{th}}$, given that the ionic strength of the electrolyte can be attributed mostly to $\mathrm{NaCl}$. Under this assumption, by using Eqs. $1-4$, the value of $Z_{\mathrm{CPE}-\mathrm{I}, \text { th }}$ at $\omega=1 \frac{\mathrm{rad}}{\mathrm{s}}$ was calculated to be $0.99 \mathrm{G} \Omega$, which is almost exactly equal to that predicted by EIS (1 G $\Omega$ ).
Therefore, the equivalent circuit models presented in this study provide a good numerical fit as well as a physiological understanding of the recorded electrochemical impedance responses of ECM-microelectrodes. This can facilitate better design and integration of ECM materials as bio-compatible encapsulations for several applications, such as, neural prosthetic devices and therapeutic applications, which typically record slow rhythm field potentials $(<200 \mathrm{~Hz})$ and action potentials $(0.1-7 \mathrm{kHz})^{1}$ as well as biosensors operating in the radio frequency spectrum $(\sim \mathrm{MHz})^{43-46}$.

\section{Enhancing biocompatibility with ECM-microelectrodes}

ECM proteins such as collagen, fibronectin, and laminin improve neuron-to-electrode surface attachment ${ }^{47}$. However, collagen IV in particular has been shown to promote neurogenesis and inhibit glial proliferation in rat cortical progenitor cell cultures derived from E16 fetuses $^{48}$. In our study, ECM-microelectrodes developed using collagen I composites with laminin, fibronectin, and collagen IV exhibited enhanced neuronal viability and superior cell adhesion and network formation in comparison to groups with collagen I microelectrodes and pre-ECM-microelectrodes. We also observed that collagen I/collagen IV microelectrodes performed best in terms of neuronal viability (Fig. 3f), cellular density on electrode surface, and expression of the axonal marker $\beta$-tubulin (Fig. 4e), thereby providing enhanced biocompatibility with neuronal environment compared with collagen I microelectrodes and pre-ECM-microelectrodes. In addition to the ECM proteins utilized in the present study, various ECM molecules exhibit functionalities in promoting neuronal cells survival and regulating various aspect of neuronal physiology. Although in the present study, only major ECM proteins are used to demonstrate the device fabrication and biocompatibility, integration of different biological and topological cues are expected to provide additional functionality for neural interfaces.

\section{Tissue responses to ECM-microelectrodes}

The in vivo histological results demonstrated that collagen I microelectrodes elicited attenuated neuroinflammation in the surrounding parenchyma compared with commercial silicon-based microelectrodes, indicating that these collagen I microelectrodes may cause significantly less tissue damage via infiltrating cells over time. Chronic microgliosis and astrogliosis can contribute to neuronal cell damage, therefore it is remarkable that these processes appeared to be limited in rodents up to 1 month following collagen I microelectrode implantation. Furthermore, reactive astrocytes can accumulate and impede the efficacy of implanted electrodes ${ }^{49,50}$. The observed reduction of gliotic pathways illustrates that the collagen I microelectrodes utilized in this study may 
possess advantageous traits that grant enhanced biocompatibility while minimizing cellular damage. Additionally, axonal processes did not appear to be structurally perturbed by the collagen I microelectrodes, suggesting that these electrodes may be implanted with minimal damage to functional neural networks. This phenomenon further indicates that collagen I microelectrodes can be assimilated along subcortical white matter tracts with minimal disruption of the surrounding neuronal processes.

\section{Recording capability of ECM-microelectrodes}

To confirm that the modulated SBF activity recorded on the collagen I microelectrodes was generated by firing of local neuronal microcircuits, we performed an additional analysis on the $\mathrm{MUA}_{\mathrm{E}}$ and compared the results to those from a control microECoG electrode placed above the brain surface, as shown in Fig. $6 \mathrm{~d}$. The $M U A_{E}$ provides an instantaneous measure of the aggregate activity of the neuronal population proximal to the electrode. Upward deflections represent increased activity ${ }^{51}$, which in this recording from the collagen I depth microelectrodes, correlated with the up states of the slow LFP oscillation (highlighted by dashed boxes in Fig. 6c). The cycle-triggered averages (CTAs) of LFP quantify the average slow oscillation profiles, which were similar between the collagen I microelectrodes and on the microECoG, as expected given the global nature of the field potential (Fig. 6d). The MUA CTAs quantify how well the local neuronal activity was correlated with the LFP oscillations. At the brain surface, the $\mathrm{MUA}_{\mathrm{E}} \mathrm{CTA}$ was relatively flat, indicating that local MUA was not modulated by the slow rhythm or, more likely, that MUA was not detectable at this site. In contrast, the MUAE CTAs of the intracortical sites show a modulation that mirrors the LFP CTAs, indicating that up/down states of each slow oscillation cycle are associated with more/less neuronal activity, respectively, as seen in Fig. 6d. Furthermore, the $\mathrm{MUA}_{\mathrm{E}} \mathrm{CTA}$ appears to have higher amplitude at the deeper intracortical site, likely reflecting the larger aggregate signal generated by infragranular pyramidal neurons. The in vivo recordings therefore confirmed the ability of collagen I microelectrodes to record both local as well as global rhythms in neuronal activities. Although only collagen I microelectrodes are used here to demonstrate the neural recording capability of the ECM-microelectrodes, due to the fact that collagen I/collagen IV microelectrodes, collagen I/laminin microelectrodes, and collagen I/fibronectin microelectrodes present similar EIS characteristics, they are expected to show similar performance for neural recordings compared with the collagen I microelectrodes. In addition to the above-mentioned ECM-microelectrodes, the microelectromechanical systems (MEMS) technologies proposed in this study will make possible a variety of ECM-microelectrodes with favorable biological properties, suitable electrochemical characteristics, as well as proper geometric and mechanical features for a broad range of neural interfacing applications.

\section{Materials and methods \\ Preparation of ECM composite films}

Four different ECM films (collagen I film, collagen I/collagen IV composite film, collagen I/fibronectin composite film, and collagen I/laminin composite film) were prepared as follows: Type I rat tail collagen in a $3 \mathrm{mg} \mathrm{mL}^{-1}$ solution (Corning ${ }^{\oplus}$, Corning, NY) was combined with $10 \times \mathrm{PBS}$ and $0.1 \mathrm{M} \mathrm{NaOH}$ at a ratio of 13:2:1 by volume, to form a base solution. To form a Type IV/ Type I collagen ECM solution, Type IV mouse collagen in a $1 \mathrm{mg} \mathrm{mL}{ }^{-1}$ solution (Corning ${ }^{\oplus}$, Corning, NY) was added to this base solution such that the total collagen content comprised 92\% Type I collagen and 8\% Type IV collagen by weight. Similarly, to form a fibronectin/Type I collagen ECM solution, fibronectin powder from rat plasma (Sigma Aldrich ${ }^{\circledast}$, St. Louis, MO) was added to the base solution such that the total protein content comprised $92 \%$ Type I collagen and $8 \%$ fibronectin by weight. Finally, to form a laminin/Type I collagen ECM solution, $6 \mathrm{mg}$ $\mathrm{mL}^{-1}$ laminin (Trevigen, Gaithersburg, MD) was added to the base solution such that the total protein content comprised $92 \%$ Type I collagen/8\% laminin by weight. In addition to these three ECM composite solutions, the base solution was used directly as a collagen I ECM solution. Each ECM solution was gently mixed until small fragments started to form. The mixed solutions were then cast into an acrylic mold, followed by polymerization at $37^{\circ} \mathrm{C}$ and $96 \%$ humidity for $24 \mathrm{~h}$ to form ECM hydrogels with fibril structures. These ECM hydrogels were then dried on glass slides in air at $37^{\circ} \mathrm{C}$ for $24 \mathrm{~h}$. The ECM films were rinsed with DI water multiple times until fully transparent films were observed. After rinsing, the films were air dried, forming uniform dried ECM films. The thickness of each film was approximately $10 \mu \mathrm{m}$.

\section{Fabrication of pre-ECM microelectrodes}

Briefly, a bare silicon wafer was first coated with Px film $(5 \mu \mathrm{m})$ using an SCS PDS 2010 Parylene coater. The electrode and conductive traces were then lithographically defined on this Px layer, as shown in Fig. 1a. Subsequently, a thin film $(100 \mathrm{~nm})$ of $\mathrm{Au}$ was deposited by e-beam evaporation and a lift-off process was performed to realize conductive Au traces. To encapsulate the device, a second layer of $\mathrm{Px}(5 \mu \mathrm{m})$ was deposited using the previously mentioned process. To interface with neurons and record their activities, both ends of the conductive Au traces, which we refer as pads hereafter, needed to be exposed. To facilitate this, first, a window type pattern was lithographically defined and registered to the Au layer. A thin film $(100 \mathrm{~nm})$ of $\mathrm{Al}$ was then deposited using e-beam 
evaporation and lifted off thereafter to remove $\mathrm{Al}$ selectively from the pads. Using the $\mathrm{Al}$ pattern as an etching mask, RIE was performed using $\mathrm{O}_{2}$ gas to (a) define the electrode footprint and (b) provide access to the pads for interfacing and recording signals. The $\mathrm{Al}$ mask was then removed via wet etching. The pre-ECM electrodes used in this study have either two or three recording sites. While the former, which possessed a smaller footprint, was used for in vivo neural recording, the latter was employed for neuronal cell studies. Pre-ECM electrodes with two recording sites had corresponding site area of $1000 \mu \mathrm{m}^{2}$ (for the site further from tip of the shank) and $2000 \mu \mathrm{m}^{2}$ (for the site closer to the tip of the shank), respectively. Also, using the same fabrication procedure, microECoG surface electrodes with recording site areas of $2500 \mu \mathrm{m}^{2}$ were fabricated as controls, to facilitate comparisons between intracortical and intracranial recordings.

\section{Fabrication of ECM microelectrodes}

The distal regions of pre-ECM depth electrodes (which ultimately will be in contact with cortical tissue) were transferred onto ECM films, as shown in Fig. 1b. Following a brief immersion in DI water, these $\mathrm{Au}-\mathrm{Px}-\mathrm{ECM}$ constructs were detached from the underlying $\mathrm{Si}$ substrate. The exposed backsides of these devices were then coated with another ECM hydrogel and air dried for $12 \mathrm{~h}$. Thus, complete encapsulation of the pre-ECM electrodes was achieved. Next, to aid the insertion process, an SS stent was laser micromachined with dimensions corresponding to the electrode footprint. The ECMencapsulated $\mathrm{Au}-\mathrm{Px}$ constructs were then hydrated and carefully placed onto this pre-patterned steel stent to ensure registration of their respective patterns. Finally, using this stent as a template, the underlying ECM films was patterned via excimer laser micro-machining to realize insertion-stent-integrated ECM-microelectrodes.

\section{Electrochemical impedance measurements}

EIS was performed in $1 \times$ PBS over a frequency range from $10 \mathrm{~Hz}$ to $1 \mathrm{MHz}$ with a potential amplitude of $20 \mathrm{mV}$. The EIS was performed using a Gamry Reference $600^{\mathrm{TM}}$ potentiostat (Gamry Instruments, Warminster, PA, United States) in a three-electrode setup where a platinum wire served as the counter electrode and $\mathrm{Ag} / \mathrm{AgCl}$ electrode as the reference electrodes. Measurements were obtained using ECM microelectrodes having two recording sites. Both sites were found to exhibit similar trends in their impedance magnitude and phase angle response, although the absolute values of these parameters were quite different. This can be mostly attributed to the differences in the areas of the recording sites. Representative results presented in this study were obtained from the larger recording site (area of $2000 \mu \mathrm{m}^{2}$ ) located closer to the tip of the electrode shank.

\section{Charge transfer resistance}

Under low-field approximation, the Butler-Volmer equation reduces to Ohm's law, which yields ${ }^{52}$

$$
J=\frac{J_{0} z F \eta}{R T},
$$

where $J$ is the current density under applied overpotential, $\eta ; J_{0}$ is the equilibrium exchange current density; $z$ is the number of electrons involved in the redox reaction; $F$ is Faraday's constant; $R$ is the gas constant; and $T$ is the temperature. Under this approximation, the charge transfer resistance, $R_{\mathrm{ct}}$, can be determined by the slope of the current vs. overpotential plot, which gives

$$
R_{\mathrm{ct}}=\frac{R T}{J_{0} z F} \text {. }
$$

\section{Simulation of $C_{\mathrm{Px}}$}

The value corresponding to $C_{\mathrm{Px}}$ was obtained from a finite element model of the pre-ECM-microelectrode in PBS solution, where the Au electrode $(20 \mu \mathrm{m}$ in width, $5 \mathrm{~mm}$ in length, and $100 \mathrm{~nm}$ in thickness; electrical conductivity, $\rho_{\mathrm{Au}}$, of $\left.0.022 \mu \Omega \mathrm{m}\right)$ and the $1 \times$ PBS solution $\left(\rho_{\text {PBS }}=1.4 \mathrm{~S} \mathrm{~m}^{-1}\right)$ occupied either sides of the Px insulation layer $(80 \mu \mathrm{m}$ in width, $5 \mathrm{~mm}$ in length, and $5 \mu \mathrm{m}$ in thickness; dielectric constant, $\varepsilon_{\mathrm{r}}$, of 3.10 ). The electrostatics model under the AC/DC module of COMSOL Multiphysics Modeling Software (COMSOL Inc., Burlington, MA, United States) was utilized to perform the simulations. The Au electrode was held at a potential of $20 \mathrm{mV}$ while the PBS domain was grounded. A relatively large PBS solution domain was chosen to capture the contributions to $C_{\mathrm{Px}}$, from fringing electric fields.

\section{Cell viability assay}

Cell viability was assessed following plating on the preECM-microelectrodes and the ECM-microelectrodes $(n=5-7$ per group) or on planar control substrates $(n=3)$ at 7 DIV as per standard protocols ${ }^{53,54}$. Briefly, neuronal cultures were rinsed with Dulbecco's phosphatebuffered saline (DPBS), incubated with ethidium homodimer-1 (EthD-1; $4 \mu \mathrm{M}$; Life technologies), and calcein AM ( $2 \mu \mathrm{M}$; Sigma) at $37^{\circ} \mathrm{C}$ for $30 \mathrm{~min}$, and then rinsed three times with DPBS. Cell viability ratio was calculated by measuring the fluorescent intensity of live cells (green) and that of the dead cells (red).

\section{Immunocytochemistry}

Neuronal adhesion, growth, and network formation were qualitatively assessed via immunocytochemistry for cultures grown on the various ECM-microelectrodes vs. the $2 \mathrm{D}$ control cultures ( $n=1$ for each group). At 7 DIV, cultures were fixed in $4 \%$ formaldehyde for $35 \mathrm{~min}$, rinsed in PBS, and permeabilized using 0.3\% Triton X100 plus $4 \%$ horse serum for $60 \mathrm{~min}$. Subsequently, mouse anti 
$\beta$-tubulin III primary antibody (Sigma-Aldrich T8578, 1:500) was added (in PBS $+4 \%$ serum) and incubated at $4{ }^{\circ} \mathrm{C}$ for $12 \mathrm{~h}$. This antibody binds to $\beta$-tubulin III protein, which is a microtubule element expressed primarily in neuronal somata and neurites. After rinsing the primary antibody, an appropriate fluorescent secondary antibody (donkey anti mouse IgG - Alexa-488 at 1:500 in PBS $+4 \%$ horse serum) was added at $18-24^{\circ} \mathrm{C}$ for $2 \mathrm{~h}$. The secondary antibody solution was then removed, and the cells were incubated with $30 \mathrm{nM}$ Hoechst in PBS for $10 \mathrm{~min}$, followed by repeated rinses with PBS. The cells were stored in PBS at $4{ }^{\circ} \mathrm{C}$ before imaging.

\section{Tissue harvest and immunohistochemistry}

At 1-month post-implant, the animals were anesthetized and transcardially perfused with heparinized saline followed by $10 \%$ formalin. The brains were removed, postfixed for $24 \mathrm{~h}$, and prepared for cryosectioning. Brains were put into $30 \%$ sucrose until saturated and frozen whole in isopentane and dry ice slurry. Frozen brains were mounted in a cryostat and faced at a plane orthogonal to the microelectrode tract and then sectioned at $35 \mu \mathrm{m}$ thick. For immunohistochemistry, sections were air-dried for $30 \mathrm{~min}$ and rehydrated in PBS three times for $5 \mathrm{~min}$ each. Next, sections were blocked with $4 \%$ normal horse serum in $0.1 \%$ Triton X/PBS for $30-45 \mathrm{~min}$. Primary antibodies were applied to the sections in $4 \%$ normal horse serum/Optimax buffer at $4{ }^{\circ} \mathrm{C}$ overnight. Sections were triple-labeled using the following primary antibodies: (1) glial-fibrillary acidic protein (GFAP), an intermediate filament protein expressed in all astrocytes that is upregulated in hypertrophic/reactive astrocytes (rabbit anti-GFAP, 1:500, Millipore Ab5804); (2) neurofilament, a cytoskeletal constituent expressed in neurons and enriched in axons (mouse anti-SMI31, 1:1000, Millipore NE1022); and (3) ionized calcium-binding adapter molecule 1 (Iba1), a marker for microglia/macrophages allowing for assessment of morphological changes associated with activation (goat anti-IBA1, Abcam, Ab5076). The next day, sections were rinsed with PBS three times for $5 \mathrm{~min}$ each. Alexa Fluor conjugated secondary antibodies (donkey anti-mouse 488, A21202; donkey anti-goat 568, A11057; donkey anti-rabbit 647, A31573; 1:1000) were applied in $4 \%$ normal horse serum/PBS for $2 \mathrm{~h}$ at room temperature. Sections were counterstained with DNA-specific fluorescent Hoechst 33342 for $5 \mathrm{~min}$ and then rinsed with PBS. After immunostaining, slides were coverslipped with Fluoromount-G mounting media.

\section{Statistical analyses}

Outlier tests were performed for all quantitative data. Data from the viability assay were analyzed using an unpaired $t$-test with Welch correction for each group against the pre-ECM group. For all statistical tests, $p<0.05$ was required for significance. Data are presented as mean \pm standard deviation.

\section{Neural recording}

Three Sprague-Dawley rats were used for the in vivo recording experiments. Each rat was anesthetized with an intraperitoneal injection of ketamine $\left(60 \mathrm{mg} \mathrm{kg}^{-1}\right)$ and dexmedetomidine $\left(0.25 \mathrm{mg} \mathrm{kg}^{-1}\right)$ and placed in a stereotaxic frame. A craniotomy was performed to expose the whisker sensory (i.e., barrel) cortex of the right hemisphere and the dura was removed. A skull screw was placed in the left parietal bone to serve as the reference electrode for the recordings. A collagen I microelectrode or a silicon microelectrode was attached to a micromanipulator and the tip of the array was implanted to a depth of $2 \mathrm{~mm}$ below the pia. In two rats, a second craniotomy exposed the barrel cortex in the opposite hemisphere and a concentric bipolar stimulating electrode was implanted at the same depth. Immediately after implantation, neural recordings were acquired with a commercial electrophysiology system (Tucker-Davis Technologies). Single pulse electrical stimuli were delivered at a rate of $0.1 \mathrm{~Hz}$ in the EP experiments. Finally, in one rat, a microECoG was placed over the exposed cortical surface and used to record cortical surface potentials. These procedures were approved by the Institutional Animal Care and Use Committee of the University of Pennsylvania.

\section{Acknowledgements}

We thank Justin C. Burrell and Kevin D. Browne for technical contributions. This work was primarily funded by the National Institutes of Health ( $\mathrm{NIH}$; R21-EB022209 (Allen)), with additional support from the NIH (U01-NS094340 (Cullen)) and the Dept. of Veterans Affairs (Merit Review Award \#B1097-I (Cullen)). Microfabrication was carried out in the Singh Center for Nanotechnology, supported in part by the US National Science Foundation National Nanotechnology Coordinated Infrastructure Program under Grant 15-42153.

\section{Authors' contributions}

W.S., A.A., and N.S. fabricated the devices; W.S., A.A., and M.R. characterized the devices and performed data analysis; S.D., L.S., and D.B. carried out in vitro and in vivo cell and tissue studies; A.R., F.V., T.L., and B.L. contributed to neural recordings and neural signal analysis; W.S., S.D., F.V., A.A., D.K.C., and M.A. cowrote the manuscript.

\section{Author details \\ ${ }^{1}$ Krishna P. Singh Center for Nanotechnology, University of Pennsylvania, Philadelphia, PA 19104, USA. ²Department of Neurosurgery, Perelman School of Medicine, University of Pennsylvania, Philadelphia, PA 19104, USA. \\ ${ }^{3}$ Department of Neurology, Perelman School of Medicine, University of Pennsylvania, Philadelphia, PA 19104, USA. ${ }^{4}$ Department of Mechanical Engineering and Applied Mechanics, School of Engineering and Applied Science, University of Pennsylvania, Philadelphia, PA 19104, USA. ${ }^{5}$ Department of Bioengineering, School of Engineering and Applied Science, University of Pennsylvania, Philadelphia, PA 19104, USA. ${ }^{6}$ Department of Electrical and Systems Engineering, School of Engineering and Applied Science, University of Pennsylvania, Philadelphia, PA 19104, USA. ${ }^{7}$ Department of Materials Science and Engineering, School of Engineering and Applied Science, University of Pennsylvania, Philadelphia, PA 19104, USA. ${ }^{8}$ Present address: Department of Mechanical and Aerospace Engineering, University of Texas at Arlington, Arlington, TX 76019, USA}

Conflict of interest

The authors declare that they have no conflict of interest. 
Supplementary information accompanies this paper at https://doi.org/ 10.1038/s41378-018-0030-5.

Received: 13 February 2018 Revised: 30 May 2018 Accepted: 5 August 2018 Published online: 24 September 2018

\section{References}

1. Fattahi, P., Yang, G., Kim, G. \& Abidian, M. R. A review of organic and inorganic biomaterials for neural interfaces. Adv. Mater. 26, 1846-1885 (2014).

2. Adewole, D. O. et al. The evolution of neuroprosthetic interfaces. Crit. Rev. Biomed. Eng. 44, 123-152 (2016).

3. Nordhausen, C. T., Maynard, E. M. \& Normann, R. A. Single unit recording capabilities of a 100 microelectrode array. Brain Res. 726, 129-140 (1996).

4. de Hemptinne, C. et al. Therapeutic deep brain stimulation reduces cortical phase-amplitude coupling in Parkinson's disease. Nat. Neurosci. 18, 779-786 (2015).

5. Hochberg, L. R. et al. Reach and grasp by people with tetraplegia using a neurally controlled robotic arm. Nature 485, 372-375 (2012).

6. Schwartz, A. B. Cortical neural prosthetics. Annu. Rev. Neurosci. 27, 487-507 (2004).

7. Bouton, C. E. et al. Restoring cortical control of functional movement in a human with quadriplegia. Nature 533, 247-250 (2016).

8. Schmidt, E. M. et al. Feasibility of a visual prosthesis for the blind based on intracortical micro stimulation of the visual cortex. Brain 119, 507-522 (1996).

9. Brumberg, J. S., Nieto-Castanon, A., Kennedy, P. R. \& Guenther, F. H. Brain-computer interfaces for speech communication. Speech Commun. 52, 367-379 (2010).

10. Bensmaia, S. J. \& Miller, L. E. Restoring sensorimotor function through intracortical interfaces: progress and looming challenges. Nat. Rev. Neurosci. 15, 313-325 (2014).

11. Barrese, J. C. et al. Failure mode analysis of silicon-based intracortical microelectrode arrays in non-human primates. J. Neural Eng. 10, 66014 (2013).

12. Bellamkonda, R. V., Pai, S. B. \& Renaud, P. Materials for neural interfaces. MRS Bull. 37, 557-561 (2012).

13. Goldstein, S. R. \& Salcman, M. Mechanical factors in the design of chronic recording intracortical microelectrodes. IEEE Trans. Biomed. Eng. 4, 260-269 (1973).

14. Hu, X., Cebe, P., Weiss, A. S., Omenetto, F. \& Kaplan, D. L. Protein-based composite materials. Mater. Today 15, 208-215 (2012).

15. Brooke, B. S., Karnik, S. K. \& Li, D. Y. Extracellular matrix in vascular morphogenesis and disease: structure versus signal. Trends Cell Biol. 13, 51-56 (2003).

16. Canver, W., Nagpal, M. L., Nachtigal, M., Borg, T. K. \& Terracio, L. Collagen expression in mechanically stimulated cardiac fibroblasts. Circ. Res. 69, 116-122 (1991).

17. Chattopadhyay, S. \& Raines, R. T. Review collagen-based biomaterials for wound healing. Biopolymers 101, 821-833 (2014).

18. Caves, J. M. et al. Elastin-like protein matrix reinforced with collagen microfibers for soft tissue repair. Biomaterials 32, 5371-5379 (2011).

19. Sottile, J. \& Hocking, D. C. Fibronectin polymerization regulates the composition and stability of extracellular matrix fibrils and cell-matrix adhesions. Mol. Biol. Cell 13, 3546-3559 (2002)

20. Novak, U. \& Kaye, A. H. Extracellular matrix and the brain: components and function. J. Clin. Neurosci. 7, 280-290 (2000).

21. Yurchenco, P. D. Basement membranes: cell scaffoldings and signaling platforms. Cold Spring Harb. Perspect. Biol. 3, a004911 (2011).

22. De Faveri, S. et al. Bio-inspired hybrid microelectrodes: a hybrid solution to improve long-term performance of chronic intracortical implants. Front. Neuroeng. 7, 7 (2014).

23. Cui, X., Wiler, J., Dzaman, M., Altschuler, R. A. \& Martin, D. C. In vivo studies of polypyrrole/peptide coated neural probes. Biomaterials 24, 777-787 (2003).

24. Jorfi, M., Skousen, J. L., Weder, C. \& Capadona, J. R. Progress towards biocompatible intracortical microelectrodes for neural interfacing applications. J. Neural Eng. 12, 11001 (2014).

25. Thelin, J. et al. Implant size and fixation mode strongly influence tissue reactions in the CNS. PLOS ONE 6, e16267 (2011).

26. Gunasekera, B., Saxena, T., Bellamkonda, R. \& Karumbaiah, L. Intracortical recording interfaces: current challenges to chronic recording function. ACS Chem. Neurosci. 6, 68-83 (2015).
27. Geckil, H., Xu, F., Zhang, X., Moon, S. \& Demirci, U. Engineering hydrogels as extracellular matrix mimics. Nanomedicine (Lond.). 5, 469-484 (2010).

28. Zorlutuna, P., Elsheikh, A. \& Hasirci, V. Nanopatterning of collagen scaffolds improve the mechanical properties of tissue engineered vascular grafts. Biomacromolecules 10, 814-821 (2009).

29. Naik, N., Caves, J., Chaikof, E. L. \& Allen, M. G. Generation of spatially aligned collagen fiber networks through microtransfer molding. Adv. Healthc. Mater. 3, 367-374 (2014).

30. Shen, W. et al. Extracellular matrix-based intracortical microelectrodes: toward a microfabricated neural interface based on natural materials. Microsyst Nanoeng. 1, 15010 (2015).

31. Kumar, V. A. et al. Microablation of collagen-based substrates for soft tissue engineering. Biomed. Mater. 9, 11002 (2014).

32. Seymour, J. P. \& Kipke, D. R. Neural probe design for reduced tissue encapsulation in CNS. Biomaterials 28, 3594-3607 (2007).

33. Shuolders, M. D. \& Raines, R. T. Collagen structure and stability. Annu. Rev. Biochem. 78, 929-958 (2010).

34. Kim, T. G., Shin, H. \& Lim, D. W. Biomimetic scaffolds for tissue engineering Adv. Funct. Mater. 22, 2446-2468 (2012).

35. Kozai, T. D. Y. et al. Ultrasmall implantable composite microelectrodes with bioactive surfaces for chronic neural interfaces. Nat. Mater. 11, 1065-1073 (2012).

36. Prasad, A. \& Sanchez, J. C. Quantifying long-term microelectrode array functionality using chronic in vivo impedance testing. J. Neural Eng. 9, 26028 (2012).

37. Blanche, T. J., Spacek, M. A. Hetke, J. F. \& Swindale, N. V. Polytrodes: high-density silicon electrode arrays for large-scale multiunit recording. J. Neurophysiol. 93, 2987-3000 (2005).

38. Cogan, S. F. Neural stimulation and recording electrodes. Annu. Rev. Biomed. Eng. 10, 275-309 (2008)

39. Shi, Y. et al. Enhanced corrosion resistance and cytocompatibility of biodegradable $\mathrm{Mg}$ alloys by introduction of $\mathrm{Mg}(\mathrm{OH}) 2$ particles into poly (L-lactic acid) coating. Sci. Rep. 7, 41796 (2017).

40. Lu, B., Zheng, S., Quach, B. Q. \& Tai, Y.-C. A study of the autofluorescence of parylene materials for $\mu$ TAS applications. Lab Chip 10, 1826-1834 (2010).

41. 1x Phosphate Buffered Saline Product Specification Sheet, Fisher Scientific (2017).

42. Bard, A. \& Faulkner, L. Electrochemical Methods. (Wiley, New York, USA, 2001).

43. Lee, H.-J. \& Yook, J.-G. Recent research trends of radio-frequency biosensors for biomolecular detection. Biosens. Bioelectron. 61, 448-459 (2014).

44. Kim, N.-Y. et al. Rapid, sensitive, and reusable detection of glucose by a robust radiofrequency integrated passive device biosensor chip. Sci. Rep. 5 (2015).

45. Chien, J. H., Chen, P. H., Kuo, L. S., Lin, C. S. \& Wang, H. Protein detection using a radio frequency biosensor with amplified gold nanoparticles. Appl. Phys. Lett. 91, 143901 (2007).

46. Huang, $X$. et al. Epidermal radio frequency electronics for wireless power transfer. Microsyst. Nanoeng. 2, 16052 (2016).

47. Slaughter, G. E., Bieberich, E., Wnek, G. E., Wynne, K. J. \& Guiseppi-Elie, A. Improving neuron-to-electrode surface attachment via alkanethiol selfassembly: an alternating current impedance study. Langmuir 20, 7189-7200 (2004).

48. Ali, S. A., Pappas, I. S. \& Parnavelas, J. G. Collagen type IV promotes the differentiation of neuronal progenitors and inhibits astroglial differentiation in cortical cell cultures. Dev. Brain Res. 110, 31-38 (1998).

49. Potter-Baker, K. A et al. A comparison of neuroinflammation to implanted microelectrodes in rat and mouse models. Biomaterials 35, 5637-5646 (2014).

50. Hirshler, Y. K., Polat, U. \& Biegon, A. Intracranial electrode implantation produces regional neuroinflammation and memory deficits in rats. Exp. Neurol. 222, 42-50 (2010).

51. Legatt, A. D., Arezzo, J. \& Vaughan, H. G. Averaged multiple unit activity as an estimate of phasic changes in local neuronal activity: effects of volumeconducted potentials. J. Neurosci. Methods 2, 203-217 (1980).

52. Franks, W., Schenker, I., Schmutz, P. \& Hierlemann, A. Impedance characterization and modeling of electrodes for biomedical applications. IEEE Trans. Biomed. Eng. 52, 1295-1302 (2005).

53. Cullen, D. K. Vernekar, V. N. \& LaPlaca, M. C. Trauma-induced plasmalemma disruptions in three-dimensional neural cultures are dependent on strain modality and rate. J. Neurotrauma 28, 2219-2233 (2011).

54. Decherchi, P., Cochard, P. \& Gauthier, P. Dual staining assessment of Schwann cell viability within whole peripheral nerves using calcein-AM and ethidium homodimer. J. Neurosci. Methods 71, 205-213 (1997). 